\title{
Stress is Associated with Severe Anti-NMDA Receptor Encephalitis: A Single-Center Retrospective Cohort Study
}

\section{Dongmei Wang}

Southern Medical University Nanfang Hospital

\section{Ni Wang}

Southern Medical University Nanfang Hospital

\section{Yue Pan}

Southern Medical University Nanfang Hospital

\section{Kaibin Huang}

Southern Medical University Nanfang Hospital

\section{Zhenzhou Lin}

Southern Medical University Nanfang Hospital

\section{Xiaomei Zhang}

Southern Medical University Nanfang Hospital

\section{Yongming Wu}

Southern Medical University Nanfang Hospital

Shengnan Wang ( $\square$ wsnpumc@smu.edu.cn )

Southern Medical University Nanfang Hospital https://orcid.org/0000-0003-4941-4071

\section{Research}

Keywords: anti-NMDAR encephalitis, stress events, teratoma, herpes virus

Posted Date: August 4th, 2021

DOI: https://doi.org/10.21203/rs.3.rs-746610/v1

License: (1) (1) This work is licensed under a Creative Commons Attribution 4.0 International License. Read Full License 


\section{Abstract}

Background: Stress was reported to trigger and exacerbate a lot of autoimmune diseases. The underlying mechanisms include disturbed hormone homeostasis, dysregulated immune function, neuroinflammation by macrophages and microglia, and so on. There have been case reports of stressinduced anti-N-methyl-d-aspartate receptor (NMDAR) encephalitis (anti-NMDARE). To elucidate the association between stress events and anti-NDMARE, we conducted a retrospective single-center cohort study.

Materials and Methods: All severe anti-NMDARE patients admitted to neuro-intensive care unit (NICU) of Nanfang Hospital between September 2013 and July 2020 were screened. The stress events within 1 year before the onset were evaluated by a questionnaire survey using the Chinese Life Event Scale. The demographic information, clinical features, laboratory results and prognosis were collected and compared between patients with and without life stress events (LSE). Patients were further classified based on the existence of teratoma and herpes viruses in cerebrospinal fluid to investigate the association between stress and anti-NMDARE.

Results: Of the 26 severe anti-NMDARE patients, 9 (34.6\%) patients reported LSE. Among them, 5 (55.6\%) had family stress, 3 (33.3\%) had work stress and $1(11.1 \%)$ had both family and work stress. No significant differences were observed in disease severity upon admission, NICU stay time, mechanical ventilation time or other laboratory results. The comorbid rate of teratoma and herpes virus was not significantly different between patients with and without LSE. However, 7 of the patients without teratoma and herpes virus $(7 / 17,41.2 \%)$ experienced LSE before the disease onset. The prognosis of patients with LSE was significantly better than that of patients without LSE $(P=0.023)$.

Conclusions: Up to $34.6 \%$ of severe anti-NMDARE patients had LSE within 1 year before the onset, which might even be underestimated. This study suggested that stress might be another risk factor for antiNMDARE besides teratoma and herpes virus. Neurologists should pay attention to the stress of the patients and provide psychological therapy to the patients.

\section{Introduction}

Anti-N-methyl-d-aspartate receptor (NMDAR) encephalitis (anti-NMDARE) was first reported in 2007. Since then, it was widely reported in young women with ovarian teratomas [1]. It is now recognized as a neuroinflammatory disease mediated by autoantibodies to the NMDAR GluN1 subunit. The antibodies crosslink NMDAR, change its surface dynamics and interaction with other synaptic proteins, inhibit receptor internalization, and lead to severe impairment of synaptic plasticity and NMDAR network function [2]. It is well known that anti-NMDARE may be associated with teratoma and herpes virus infection [3], and also, there are reports in the literature that stress can induce anti-NMDARE $[4,5]$.

Stress occurs when people's needs exceed their psychosocial resources or adaptability. Some acute life events, such as the death of relatives, long-term work challenges, can be a source of demand or stress [6]. 
There is now a lot of convincing evidence that acute stress can stimulate plasma inflammatory response. Life stress events (LSE) have widely reported to be associated with the severity and progression of many diseases, including depression, cardiovascular disease, human immunodeficiency virus /acquired immunodeficiency syndrome infection, chronic obstructive pulmonary disease, breast cancer, asthma, toxic diffuse goiter, and autoimmune diseases [7-15].

There has been a case reported that emotional stress-related factors induce anti-NMDARE [4]. To evaluate the correlation between stress and severe anti-NMDARE, and to elucidate the clinical features of severe anti-NMDARE with LSE, we conducted this retrospective single center cohort study.

\section{Materials And Methods}

\section{Study design and participants}

We screened all patients diagnosed with anti-NMDARE who were admitted to the neuro-intensive care unit (NICU) of Nanfang Hospital, a university-affiliated academic hospital, between September 2013 and July 2020. The diagnostic criteria for anti-NMDARE were as follows: rapid onset (less than 3 months) of one or more of the six major groups of symptoms, including abnormal (psychiatric) behavior or cognitive dysfunction, speech dysfunction (pressured speech, verbal reduction, and mutism), seizures, movement disorders (dyskinesias or rigidity/abnormal postures), decreased level of consciousness, and autonomic dysfunction or central hypoventilation; the presence of anti-NMDAR antibodies in cerebrospinal fluid (CSF); reasonable exclusion of other disorders [16]. Severe anti-NMDARE was defined as fulfilling one or more of following criteria: respiratory failure requiring endotracheal intubation and/or mechanical ventilation (MV); disturbance of consciousness; status epilepticus [17]. Exclusion criteria included: secondary anti-NMDARE; history of psychological or psychiatric illness; loss to follow-up.

A questionnaire survey about the patients was conducted on the family members upon admission and a retrospective survey was conducted on the patients themselves after recovery using the Chinese Life Event Scale (LES). LES was compiled by Yang et. al. according to China's national conditions. There are a total of 50 items, of which 48 are mainly family life items (28), work or study items (13) and social life items (7). These are three common life events in our country. The other two blank items are filled in by the subjects according to their personal circumstances [18]. Each item got one point and the sum of the scores was defined as LSE score. If a patient or family member reported a positive event within one year before the onset of the disease, the patient was defined as LSE+.

This study was approved by the Ethics Committee of Nanfang Hospital, Southern Medical University. Informed consent was waived by the review board because this study was observational and retrospective, and all data were fully de-identified.

\section{Data collection}


Electrical medical records were carefully reviewed to collect the patients' information $\nabla$ including demographic information, teratomas, Glasgow coma scale (GCS) score on admission, Acute Physiology and Chronic Health Evaluation (APACHE) II score on admission, laboratory results, immunotherapy protocol (steroids, intravenous immunoglobulins (IVIG), plasma exchange (PE)), LSE scores, length of NICU stay, duration of MV, and the modified Rankin Scale (mRS) score of 6-month after discharge. Information on functional status after discharge was obtained through telephone interview or clinic interview by a trained neurologist blinded to the study data. We defined mRS $\leq 2$ as a good prognosis, while $\mathrm{mRS} \geq 3$ as a poor prognosis.

\section{Statistical analysis}

Student's t-test was used to compare data for continuous variables. Mann-Whitney and Chi-Squared tests were used to compare data for non-continuous and categorical variables between the two groups. A pvalue $<0.05$ was considered statistically significant. SPSS statistical software v. 22 was used for the statistically analysis.

\section{Results}

Of 29 patients screened for eligibility, 26 fulfilled the inclusion and exclusion criteria (Fig. 1). In this cohort, 9 patients $(9 / 26,34.6 \%)$ were reported to have experienced LSE within one year before the disease onset. In the LSE+ group, 5 patients reported family stress (55.6\%), 3 patients reported work stress (33.3\%) and 1 patient reported stress from both family and work (11.1\%). Seven patients $(77.8 \%)$ had an LSE score of 1 , one patient $(11.1 \%)$ had a score of 2 , and one patient $(11.1 \%)$ had a score of 3.

The clinical characteristics were compared between the patients with and without LSE (Table 1). There were no significant differences between the two groups in terms of demographic characteristics, APACHE II score, GCS score, teratoma incidence, NICU stay, mechanical ventilation time and laboratory results of complete blood count (CBC) and CSF results. One LSE+ patient had an extremely long NICU stay [19], and she was excluded from the analysis of NICU stay and MV time to avoid bias.

The association between teratomas, herpes virus and LSE was shown in Table 2. There was no significant difference in the incidence of teratoma between the two groups $(P=0.357)$, though the proportion seemed lower in LSE+ group $(1 / 9,11.1 \%)$ than that $(6 / 17,35.3 \%)$ in LSE- group. And also, the comorbidities of herpes virus detection revealed no significant difference between the two groups $(P=1.000)$. Comorbidities of teratomas or herpes virus revealed no significant difference between the two groups $(P=0.418)$. It is worth noting that among patients without teratoma and herpes virus, $7(7 / 17$, $41.2 \%)$ had LSE.

All the patients received first-line treatment, including teratoma resection if exists, steroids, IVIG, and PE. There was no significant difference in treatment strategies between the two groups. During follow-up, 18 patients $(18 / 26,69.2 \%)$ achieved a good prognosis. In the LSE+ group, all patients (100\%) achieved a 
good prognosis, while only $52.9 \%$ of LSE- patients achieved a good prognosis. The prognosis of LSE+ patients was significantly better than that of patients without LSE $(P=0.023)$.

\section{Discussion}

In this retrospective cohort study, we found that in severe anti-NMDARE patients, as high as $34.6 \%$ patients had experienced LSE within one year before the disease onset. Since some patients could not provide information by themselves, this incidence could still be underestimated. Patients with LSE seemed to achieve a better prognosis than those without LSE.

Stress has been reported to trigger and exacerbate a lot of autoimmune diseases, such as rheumatoid arthritis, systemic lupus erythematosus, inflammatory bowel disease, multiple sclerosis, Graves' disease as well as other autoimmune conditions [20]. And also patients with stress-related disorders were at increased risk of autoimmune disease $[20,21]$. A large number of studies have tried to elucidate the underlying mechanisms of stress and various autoimmune diseases. The suggested hypotheses included disturbed homeostasis, neuroinflammation by macrophages and microglia, and evoked humoral immune response $[20,22]$.

Rare studies have reported the association between stress and anti-NMDARE, and the mechanism was still unclear $[5,23,24]$. Obi CA and their colleagues suggested that emotional stress triggered immune dysregulation, which ultimately resulted in anti-NMDARE [4]. The other suggested mechanism was the preexisting anti-NMDAR antibody in the peripheral blood $[25,26]$. Under some stress inducers, the integrity of blood-brain barrier (BBB) was disrupted and the antibody was passed through the barrier, causing the disease [5]. In animal models, restraint stress mediates time-dependent alterations in the permeability of the BBB [27]. Acute psychosocial stress also has proinflammatory effects mediated by activation of mast cells and is associated with BBB opening [28]. Maladaptation of the BBB to persisting and/or severe stressors may contribute to detrimental health outcomes [29]. In this study, we found that as high as $34.6 \%$ of severe anti-NMDARE patients reported LSE before the disease onset, which could still be underestimated. We have no idea whether the disease was attributed to the stress induced immunedysregulation or stress induced BBB leakage with preexisting serum antibody. However, we did find an association between stress and severe anti-NMDARE clinically, and more research was warranted to investigate the exact mechanism.

There was no significant difference in the comorbidities of teratoma and herpes virus between LSE + and LSE- groups. Nevertheless, $41.2 \%$ of the patients without teratoma and herpes virus experienced LSE before disease onset, which indicated that stress might be another risk factor for anti-NMDARE besides teratomas and herpes virus. For anti-NMDARE patients, psychological therapy and stress management should be considered to prevent stress-induced immune dysregulation, as this might help prevent relapse.

Another interesting phenomenon in this study was that patients with LSE revealed a better prognosis although there was no significant difference in disease severity at admission. The underlying reasons were unclear. The relatively low incidence of teratoma in the LSE + group was a possible reason, avoiding 
with surgery damage. The second possible reason could be that for most patients, stress was discontinued after the diseases since most patients were unconscious. Another possible reason was that patients with poor prognosis could not provide LSE information by themselves, and the LSE survey was conducted on their family members, which could cause bias. Therefore, this prognosis result should be interpreted with caution and more studies are needed to elucidate the problem.

This study has some limitations. First, this is a single-center retrospective study with a very limited sample size. Secondly, this study only focused on severe patients. Third, the stress assessment was recalled by patients and/or family members, which might introduce bias. Due to all the limitations, some results of the study should be interpreted with caution and more research is required.

\section{Conclusions}

In this study, we found that $34.6 \%$ of severe anti-NMDARE patients had stress events within one year before the onset of the disease, which might be still underestimated. As high as $41.2 \%$ of the patients without teratoma and herpes virus experienced LSE before the disease onset, which suggested that stress might be another risk factor for anti-NMDARE in addition to teratomas and herpes virus. Neurologists should pay attention to the stress events of anti-NMDARE patients, and psychological therapy should be considered during maintenance therapy.

\section{Abbreviations}

anti-NMDAR: anti-N-methyl-d-aspartate receptor; anti-NMDARE: anti-N-methyl-d-aspartate receptor encephalitis; APACHE: Acute Physiology and Chronic Health Evaluation; BBB: blood-brain barrier; CBC: complete blood count; CSF: cerebrospinal fluid; GCS: Glasgow coma scale; IVIG: intravenous immunoglobulins; LES: Life Event Scale; LSE: Life stress events; mRS: modified Rankin Scale; MV: mechanical ventilation; NICU: neuro-intensive care unit; PE: plasma exchange

\section{Declarations}

Acknowledgements: The authors thank all the patients and their family members, for their generosity and cooperation in this study.

\section{Authors' contributions:}

DW, YW, and SW are responsible for concepts and design. $\mathrm{KH}, \mathrm{ZL}$ and $\mathrm{XZ}$ are are responsible for data collecting. NW and YP are responsible for statistical analysis and result interpretation. All authors contributed to the article and approved the submitted version. All authors acquired, analyzed, and interpreted the data. The manuscript was prepared by DW and NW.

\section{Funding}


This study was supported by President Foundation of Nanfang Hospital, Southern Medical University (No.2020B006, 2019B007).

Availability of data and materials

The data of the study are available from the corresponding author on reasonable request.

\section{Ethics approval and consent to participate}

This study was approved by the Ethics Committee of Nanfang Hospital, Southern Medical University. Informed consent was waived by the review board because this study was observational and retrospective, and all data were fully de-identified.

Consent for publication

Not applicable

\section{Competing interests}

The authors declare no competing interests.

\section{References}

1. Dalmau J, Tüzün E, Wu H-y, Masjuan J, Rossi JE, Voloschin A, Baehring JM, Shimazaki H, Koide R, King D, et al: Paraneoplastic anti-N-methyl-D-aspartate receptor encephalitis associated with ovarian teratoma. Annals of Neurology 2007, 61:25-36.

2. Dalmau J, Armangué T, Planagumà J, Radosevic M, Mannara F, Leypoldt F, Geis $C$, Lancaster $E$, Titulaer MJ, Rosenfeld MR, Graus F: An update on anti-NMDA receptor encephalitis for neurologists and psychiatrists: mechanisms and models. The Lancet Neurology 2019, 18:1045-1057.

3. DeSena A, Graves D, Warnack W, Greenberg BM: Herpes Simplex Encephalitis as a Potential Cause of Anti-N-Methyl-d-Aspartate Receptor Antibody Encephalitis. JAMA Neurology 2014, 71:344.

4. Obi CA, Thompson E, Mordukhaev L, Khan I, Zhang NJ: Anti-N-methyl-d-aspartate receptor encephalitis triggered by emotional stress. Baylor University Medical Center Proceedings 2019, 32:605-606.

5. Vahabi Z, Etesam F, Zandifar A, Badrfam R: Psychosocial stress, blood brain barrier and the development of anti N-methyl-D-aspartate receptor (NMDAR) encephalitis. Multiple Sclerosis and Related Disorders 2021, 50:102876.

6. Steptoe A, Kivimäki M: Stress and cardiovascular disease. Nature Reviews Cardiology 2012, 9:360370.

7. Cohen S, Murphy MLM, Prather AA: Ten Surprising Facts About Stressful Life Events and Disease Risk. Annual Review of Psychology 2019, 70:577-597. 
8. Chilunga FP, Boateng D, Henneman P, Beune E, Requena-Mendez A, Meeks K, Smeeth L, Addo J, Bahendeka S, Danquah I, et al: Perceived discrimination and stressful life events are associated with cardiovascular risk score in migrant and non-migrant populations: The RODAM study. Int J Cardiol 2019, 286:169-174.

9. Kendler KS, Karkowski LM, Prescott CA: Causal relationship between stressful life events and the onset of major depression. Am J Psychiatry 1999, 156:837-841.

10. Lu Y, Nyunt MS, Gwee X, Feng L, Feng L, Kua EH, Kumar R, Ng TP: Life event stress and chronic obstructive pulmonary disease (COPD): associations with mental well-being and quality of life in a population-based study. BMJ Open 2012, 2.

11. Bahri N, Fathi Najafi T, Homaei Shandiz F, Tohidinik HR, Khajavi A: The relation between stressful life events and breast cancer: a systematic review and meta-analysis of cohort studies. Breast Cancer Res Treat 2019, 176:53-61.

12. Pence BW, Raper JL, Reif S, Thielman NM, Leserman J, Mugavero MJ: Incident stressful and traumatic life events and human immunodeficiency virus sexual transmission risk behaviors in a longitudinal, multisite cohort study. Psychosom Med 2010, 72:720-726.

13. Lietzen R, Virtanen P, Kivimaki M, Sillanmaki L, Vahtera J, Koskenvuo M: Stressful life events and the onset of asthma. Eur Respir J 2011, 37:1360-1365.

14. Porcelli B, Pozza A, Bizzaro N, Fagiolini A, Costantini M-C, Terzuoli L, Ferretti F: Association between stressful life events and autoimmune diseases: A systematic review and meta-analysis of retrospective case-control studies. Autoimmunity Reviews 2016, 15:325-334.

15. Winsa B, Adami HO, Bergström R, Gamstedt A, Dahlberg PA, Adamson U, Jansson R, Karlsson A: Stressful life events and Graves' disease. Lancet 1991, 338:1475-1479.

16. Xu X, Lu Q, Huang Y, Fan S, Zhou L, Yuan J, Yang X, Ren H, Sun D, Dai Y, et al: Anti-NMDAR encephalitis: A single-center, longitudinal study in China. Neurol Neuroimmunol Neuroinflamm 2020, 7.

17. Wang D, Su S, Tan M, Wu Y, Wang S: Paroxysmal Sympathetic Hyperactivity in Severe Anti-N-Methyld-Aspartate Receptor Encephalitis: A Single Center Retrospective Observational Study. Front Immunol 2021, 12.

18. D Y, Y Z: Life Event Scale.Mental Health Assessment Scale Manual. Chin Ment Health J 1993, 3:3941.

19. Wang D, Wu Y, Ji Z, Wang S, Xu Y, Huang K, Peng Y, Zheng H, Wang H, Zhang X, Pan S: A refractory anti-NMDA receptor encephalitis successfully treated by bilateral salpingo-oophorectomy and intrathecal injection of methotrexate and dexamethasone: a case report. Journal of International Medical Research 2020, 48:030006052092566.

20. Sharif K, Watad A, Coplan L, Lichtbroun B, Krosser A, Lichtbroun M, Bragazzi NL, Amital H, Afek A, Shoenfeld Y: The role of stress in the mosaic of autoimmunity: An overlooked association. Autoimmunity Reviews 2018, 17:967-983. 
21. Song H, Fang F, Tomasson G, Arnberg FK, Mataix-Cols D, Fernández de la Cruz L, Almqvist C, Fall K, Valdimarsdóttir UA: Association of Stress-Related Disorders With Subsequent Autoimmune Disease. Jama 2018, 319:2388.

22. Marsland AL, Walsh C, Lockwood K, John-Henderson NA: The effects of acute psychological stress on circulating and stimulated inflammatory markers: A systematic review and meta-analysis. Brain, Behavior, and Immunity 2017, 64:208-219.

23. Pan H, Oliveira B, Saher G, Dere E, Tapken D, Mitjans M, Seidel J, Wesolowski J, Wakhloo D, KleinSchmidt C, et al: Uncoupling the widespread occurrence of anti-NMDAR1 autoantibodies from neuropsychiatric disease in a novel autoimmune model. Molecular Psychiatry 2018, 24:1489-1501.

24. Pan H, Steixner-Kumar AA, Seelbach A, Deutsch N, Ronnenberg A, Tapken D, von Ahsen N, Mitjans M, Worthmann $\mathrm{H}$, Trippe $\mathrm{R}$, et al: Multiple inducers and novel roles of autoantibodies against the obligatory NMDAR subunit NR1: a translational study from chronic life stress to brain injury. Molecular Psychiatry 2020.

25. Zerche M, Weissenborn K, Ott C, Dere E, Asif AR, Worthmann H, Hassouna I, Rentzsch K, Tryc AB, Dahm L, et al: Preexisting Serum Autoantibodies Against the NMDAR Subunit NR1 Modulate Evolution of Lesion Size in Acute Ischemic Stroke. Stroke 2015, 46:1180-1186.

26. Hammer C, Stepniak B, Schneider A, Papiol S, Tantra M, Begemann M, Sirén AL, Pardo LA, Sperling S, Mohd Jofrry S, et al: Neuropsychiatric disease relevance of circulating anti-NMDA receptor autoantibodies depends on blood-brain barrier integrity. Molecular Psychiatry 2013, 19:1143-1149.

27. Xu G, Li Y, Ma C, Wang C, Sun Z, Shen Y, Liu L, Li S, Zhang X, Cong B: Restraint Stress Induced Hyperpermeability and Damage of the Blood-Brain Barrier in the Amygdala of Adult Rats. Frontiers in Molecular Neuroscience 2019, 12.

28. Hendriksen E, van Bergeijk D, Oosting RS, Redegeld FA: Mast cells in neuroinflammation and brain disorders. Neuroscience \& Biobehavioral Reviews 2017, 79:119-133.

29. Segarra M, Aburto MR, Acker-Palmer A: Blood-Brain Barrier Dynamics to Maintain Brain Homeostasis. Trends in Neurosciences 2021, 44:393-405.

\section{Tables}

Table 1 The clinical features of the patients 


\begin{tabular}{|c|c|c|c|}
\hline & $\begin{array}{l}\text { LSE+ } \\
(n=9)\end{array}$ & $\begin{array}{l}\text { LSE- } \\
\qquad(n=17)\end{array}$ & $p$ \\
\hline Gender (female), n (\%) & $6(66.7 \%)$ & $12(70.6 \%)$ & $1.0^{\mathrm{c}}$ \\
\hline Age (years), mean $\pm S D$ & $28.67 \pm 11.09$ & $26.35 \pm 10.40$ & $0.603^{a}$ \\
\hline *Time of NICU stay (days), median (IQR) & $\begin{array}{l}23.5(0.75 \\
36.25)\end{array}$ & $25(7.5,68.5)$ & $0.771^{b}$ \\
\hline $\begin{array}{l}\text { *Mechanical ventilation time (hours), median } \\
(\text { IQR) }\end{array}$ & $26(0,336)$ & $17(0,432)$ & $0.734^{b}$ \\
\hline 6-month mRS $\leq 2, \mathrm{n}(\%)$ & $9(100 \% \rrbracket$ & $9(52.9 \%)$ & $0.023^{c}$ \\
\hline Teratoma, n (\%) & $1(11.1 \%)$ & $6(35.3 \%)$ & $0.357^{c}$ \\
\hline \multicolumn{4}{|l|}{ First-line therapy, n (\%) } \\
\hline Steroids & $7(77.7 \%)$ & $11(64.7 \%)$ & $0.667^{c}$ \\
\hline IVIG & $7(77.7 \%)$ & $16(94.1 \%)$ & $0.268^{c}$ \\
\hline PE & $7(77.7 \%)$ & $14(82.4 \%)$ & $1.0^{\mathrm{C}}$ \\
\hline \multicolumn{4}{|l|}{ CSF results } \\
\hline WBC (cells/ $\mu \mathrm{L})$, median (IQR) & $2(0,36.5)$ & $4(0,45)$ & $0.442^{b}$ \\
\hline Total protein (g/L), median (IQR) & $0.21(0.15,0.28)$ & $\begin{array}{l}0.23(0.17 \\
0.295)\end{array}$ & $0.787^{b}$ \\
\hline Glucose (mmol/L), median (IQR) & $3.43(3.01,4.22)$ & $3.9(3.60,4.39)$ & $0.235^{b}$ \\
\hline CSF herpes virus detection, $\mathrm{n}(\%)$ & $1(11.1 \%)$ & $2(11.8 \%)$ & $1.0^{c}$ \\
\hline \multicolumn{4}{|l|}{$\mathrm{CBC}$ results } \\
\hline WBC (G/L), mean $\pm S D$ & $10.4 \pm 3.57$ & $10.0 \pm 3.82$ & $0.800^{a}$ \\
\hline Lymphocytes (G/L), mean $\pm S D$ & $1.39 \pm 0.52$ & $1.53 \pm 0.77$ & $0.621^{a}$ \\
\hline Neutrophils (G/L), mean $\pm S D$ & $8.37 \pm 3.25$ & $7.62 \pm 4.06$ & $0.639^{a}$ \\
\hline
\end{tabular}

${ }^{\text {a }}$, Student's t-test; ${ }^{\mathrm{b}}$, Mann-Whitney test; ${ }^{\text {c: }}$. Chi-square test.

* Exclude one patient for her extremely long hospital stay and NICU stay.

Abbreviations: CBC, complete blood count; CSF, cerebrospinal fluid; IQR, interquartile range; IVIG, intravenous immunoglobulins; LSE, life stress events; mRS, modified Rankin Scale; PE, plasma exchange; 
WBC, white blood cell.

Table 2: The association between teratomas, CSF herpes virus and LSE in the patients

\begin{tabular}{|c|c|c|c|c|}
\hline & & \multicolumn{2}{|c|}{ LSE, $n$} & \multirow{2}{*}{$P^{*}$} \\
\hline & & Yes & No & \\
\hline \multirow[t]{2}{*}{ Teratomas } & Yes & 1 & 6 & \multirow[t]{2}{*}{0.357} \\
\hline & No & 8 & 11 & \\
\hline \multirow[t]{2}{*}{ CSF herpes virus } & Yes & 1 & 2 & \multirow[t]{2}{*}{1.000} \\
\hline & No & 8 & 15 & \\
\hline \multirow[t]{2}{*}{ Teratomas or CSF herpes virus } & Yes & 2 & 7 & \multirow[t]{2}{*}{0.418} \\
\hline & No & 7 & 10 & \\
\hline
\end{tabular}

${ }^{*}$, Chi-square test;

Abbreviations: CSF, cerebrospinal fluid; LSE, life stress events.

\section{Figures}

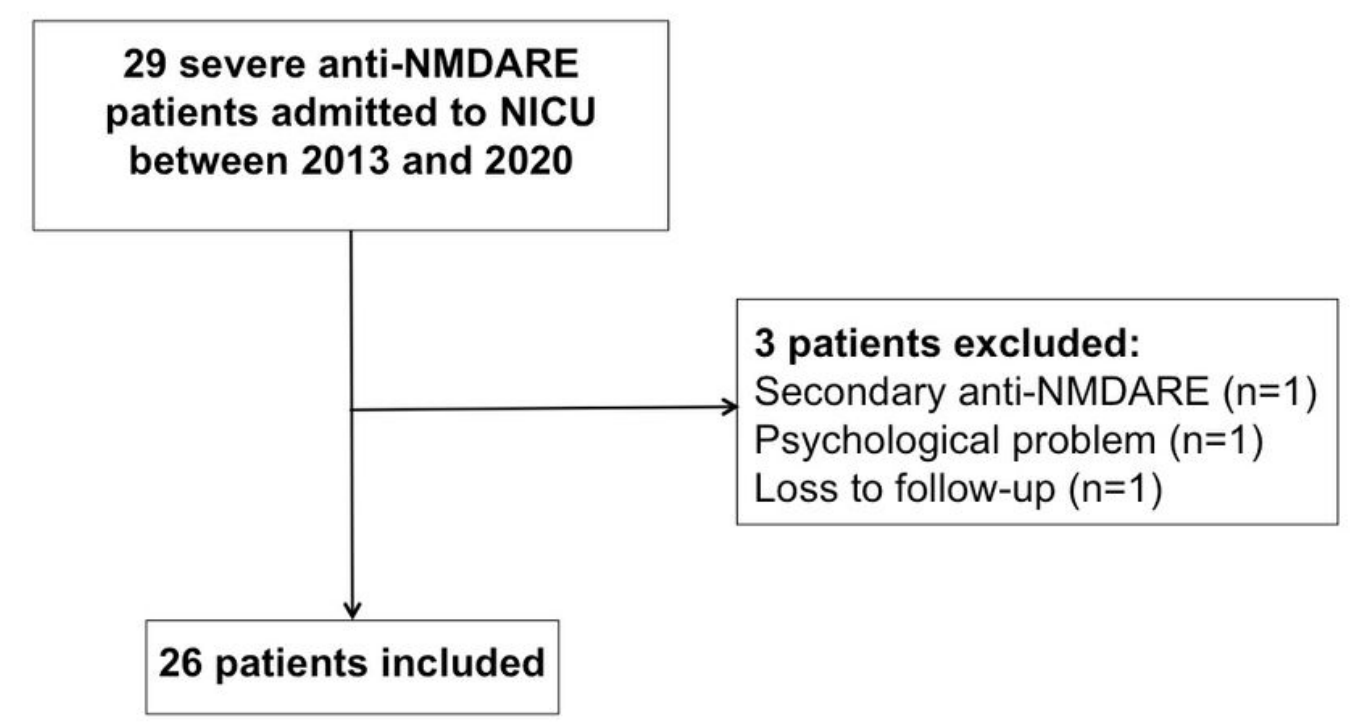




\section{Figure 1}

Flow chart of enrolled patients. anti-NMDARE, anti-N-methyl-d-aspartate receptor encephalitis; NICU, neuro-intensive care unit. 